\title{
The MICE beam line instrumentation (trackers and PID) for precise emittance measurement.
}

\author{
F.J.P. Soler ${ }^{* \dagger}$ \\ University of Glasgow \\ E-mail: paul.solereglasgow.ac.uk
}

The International Muon Ionization Cooling Experiment (MICE) will carry out a systematic investigation of ionization cooling of a muon beam, for the future Neutrino Factory and the Muon Collider. As the emittance measurement will be done on a particle-by-particle basis, a sophisticated beam instrumentation is needed to measure both particle coordinates and timing versus Radio Frequency (RF) phase in a harsh environment due to high particle rates, fringe magnetic fields and RF backgrounds. A Particle Identification (PID) system, based on three time-of-flight stations (with resolutions up to 50-60 ps), two aerogel Cherenkov counters and a KLOE-like calorimeter (KL), has been constructed and has allowed the commissioning of the MICE muon beam line in 2010. It will be soon followed by an Electron Muon Ranger to determine the muon range at the apparatus downstream-end and later by two tracker detectors to trace incoming particles inside two high-field superconducting solenoids. Detector performances will be shown and their use for the beam line characterization will be fully illustrated.

36th International Conference on High Energy Physics

4-11 July 2012

Melbourne, Australia

\footnotetext{
*Speaker.

On behalf of the MICE Collaboration.
} 


\section{Introduction}

The Neutrino Factory produces beams of neutrinos from the decay of muons for long-baseline neutrino oscillation experiments [1]. The Neutrino Factory has been optimised for the recently measured value of $\sin ^{2} 2 \theta_{13} \sim 0.097[2,3,4,5,6]$. A $10 \mathrm{GeV} / \mathrm{c}$ muon beam with a $100 \mathrm{kton}$ Magnetised Iron Neutrino Detector (MIND) at a distance between 2000 and $2500 \mathrm{~km}$ offers the best CP violation discovery sensitivity $\left(\Delta \delta_{C P} \sim 5^{\circ}\right)$ out of all future facilities [7]. In order to achieve the design goal of $10^{21}$ muons per year, the transverse emittance of the muon beam needs to be reduced from $18 \mathrm{~mm}$ rad down to $7.5 \mathrm{~mm}$ rad in a $75 \mathrm{~m}$ long solenoid focused ionization cooling channel with 201.25 MHz RF cavities and lithium hydride ( $\mathrm{LiH}$ ) absorbers. Furthermore, a Neutrino Factory is the first step towards a multi-TeV Muon Collider, which requires 6D ionization cooling, with emittance reduction of $10^{6}$ [8].

The Muon Ionization Cooling Experiment (MICE) is being carried out at the Rutherford Appletion Laboratory (RAL) in the UK, to demonstrate ionization cooling. Muon cooling is achieved with three low density absorbers (liquid hydrogen or $\mathrm{LiH}$ ) inside focusing magnetic fields, and eight $201 \mathrm{MHz}$ RF cavities enclosed by two coupling coils to restore the longitudinal momentum. The change in emittance $\varepsilon$ is:

$$
\frac{d \varepsilon}{d z}=-\frac{\varepsilon}{E_{\mu} \beta^{2}} \frac{d E_{\mu}}{d z}+\frac{\beta_{\perp}}{2 m \beta^{3}} \frac{(13.6 \mathrm{MeV})^{2}}{E_{\mu} X_{0}},
$$

where $\beta=v / c, E_{\mu}$ and $m$ are the muon energy and mass, $\beta_{\perp}$ is the Twiss transverse beta function and $X_{0}$ is the radiation length. The best cooling is achieved with a low- $Z$ absorber, such as liquid hydrogen or $\mathrm{LiH}$. The aim of MICE is to measure $\sim 10 \%$ emittance reduction for muon beams with emittance between 2 and $10 \mathrm{~mm}$ rad and momenta $140-240 \mathrm{MeV} / \mathrm{c}$ with $1 \%$ precision $\left(\Delta \varepsilon / \varepsilon_{\text {in }}=\right.$ $\left.10^{-3}\right)$. Hence, MICE is a single particle muon spectrometer to achieve the required precision.

The MICE programme is being implemented in steps. Step I, consisting of the muon beam and beam line detectors, has already been completed. Step IV, consisting of the liquid hydrogen absorber inside a focus-coil magnet and two spectrometer solenoids, is scheduled by 2014. The final Step VI, with three Absorber Focus Coil (AFC) modules and two RF-Coupling Coil (RFCC) modules with four $201 \mathrm{MHz}$ RF cavities per module (Figure 1), is scheduled by 2016.

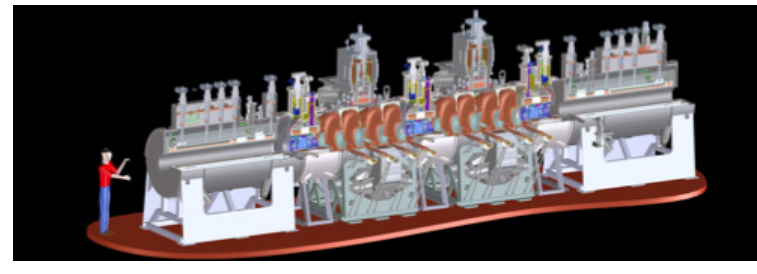

Figure 1: Three dimensional cutaway rendering of MICE.

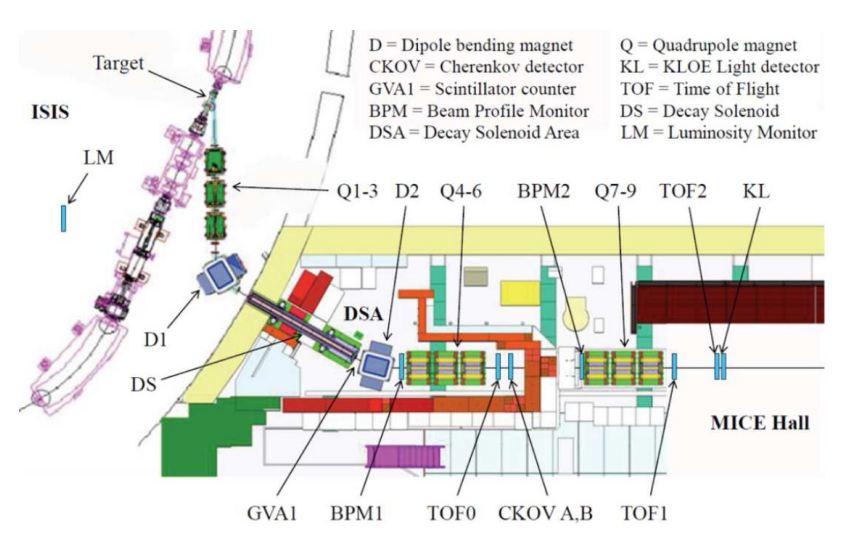

Figure 2: Schematic view of the MICE beam. 


\section{MICE beam line}

The MICE muon beam, used for Step I operations during 2010 and 2011, is derived from the ISIS synchrotron at RAL in Oxfordshire, UK. The $800 \mathrm{MeV}$ protons impinge on a dipping titanium target at a rate of $\sim 1 \mathrm{~Hz}$. The MICE beam is shown in Figure 2 and consists of an extraction region with three quadrupole magnets (Q1-Q3), a dipole bending magnet (D1) for selection of pions with a determined momentum, a pion decay region consisting of a superconducting solenoidal magnet, operating at a temperature of $4.5 \mathrm{~K}$ with a nominal magnetic solenoid field of $5 \mathrm{~T}$ (DS), a second dipole magnet for muon momentum selection (D2), the muon matching section with two quadrupole triplets (Q4-Q9), three Time-of-Flight counters (TOF0, TOF1 and TOF2) and two Cherenkov modules (CKOVA and CKOVB). There is an additional scintillator counter (named GVA1) upstream of the second dipole and the Beam Profile Monitor (BPM1) downstream of the second dipole, which were used for particle rate and position monitoring.

\section{MICE detectors}

\subsection{Time of Flight and Cherenkov detectors}

The three TOF counters (TOF0, TOF1 and TOF2) have the double function of performing particle identification and measuring the RF phase. A precision in the RF phase of $50^{\circ}$ at $201 \mathrm{MHz}$, implies a TOF timing resolution of $\sim 70 \mathrm{ps}$. To achieve a $99 \%$ rejection of pion contamination in the muon beam, a resolution of $\sim 100$ ps between TOF0 and TOF1 is needed. Therefore, the required TOF resolution is between 50 and 60 ps for each TOF station.

All the TOF stations use 1 inch thick Bicron BC404 or BC420 scintillator counters oriented along the $x$ and $y$ directions, read out at both ends by fast Hamamatsu R4998 photomultipliers (PMT). The dimensions of TOF0 are $40 \times 40 \mathrm{~cm}^{2}$ with 10 scintillators of $4 \mathrm{~cm}$ width per plane (Figure 3) . Both TOF1 and TOF2 use $6 \mathrm{~cm}$ wide scintillators, with TOF1 covering an area $42 \times$ $42 \mathrm{~cm}^{2}$ and TOF2 an area of $60 \times 60 \mathrm{~cm}^{2}$. The PMTs need to operate in a magnetic field with $B_{\|} \sim 200-300 \mathrm{G}$ and $B_{\perp} \sim 1 \mathrm{kG}$, requiring an external field cage surrounding TOF1 and ARMCO shielding around the PMTs of TOF2 to attenuate the magnetic field. The PMT signals, after a splitter, are sent to Lecroy 4415 leading edge discriminators and then to CAEN V1290 TDC and a CAEN V1724 flash ADC for time-walk corrections. The TOF0 time resolution is 51 ps, TOF1 is $53 \mathrm{ps}$ and TOF2 is 52 ps [9]. Figure 5 shows the time of flight between TOF0 and TOF1, with clear peaks for electrons (26 ns), muons (29 ns) and pions (31 ns).

Further pion-muon separation between 240 and $300 \mathrm{MeV} / \mathrm{c}$ is achieved with two Cherenkov detectors, placed behind TOF0 (Figure 3). The aerogels for the two Cherenkov counters CKOVa and CKOVb (Figure 4) have indices of refraction $n_{a}=1.07$ and $n_{b}=1.12$, corresponding to densities $\rho_{a}=0.225 \mathrm{~g} \mathrm{~cm}^{-3}$ and $\rho_{b}=0.370 \mathrm{~g} \mathrm{~cm}^{-3}$ [10]. The aerogel tiles are two layers thick (2.3 $\mathrm{cm}$ ) and cover a $46 \times 46 \mathrm{~cm}^{2}$ area. Four 8 inch EMI 9356KB PMTs collect the Cherenkov light, read out by a high frequency sampling digitiser CAEN V1731 (1 GS/s maximum sampling rate).

\subsection{Calorimeters (KL and EMR)}

The MICE KLOE-Like (KL) lead-scintillating fibre preshower detector is dedicated to electronmuon separation downstream. It consists of $1 \mathrm{~mm}$ diameter blue scintillating fibres glued between 


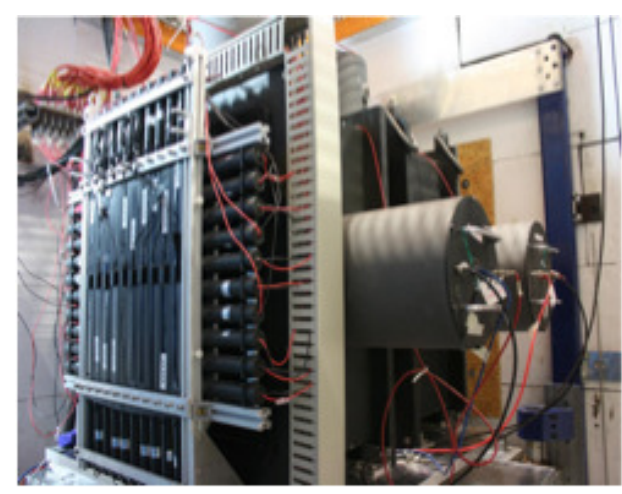

Figure 3: TOF0 and the two Cherenkov detectors.

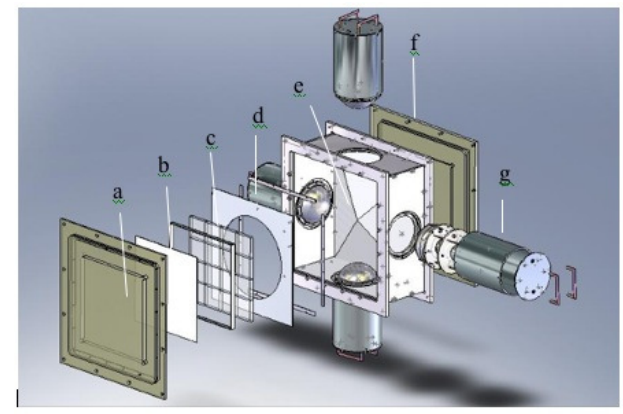

Figure 4: Cutaway schematic of Cherenkov counter: a) entrance window, b) mirror, c) aerogel mosaic, d) acetate window, e) GORE-TEX reflector panel, f) exit window, g) 8 inch PMT in iron shield.

$0.3 \mathrm{~mm}$ thick grooved lead plates, similar to the KLOE calorimeter [11]. The active volume is $93 \times 4 \times 93 \mathrm{~cm}^{3}$, divided into 7 modules supported by an iron frame, which also acts as a magnetic shield. The KL has a thickness of $2.5 X_{0}$ and $0.15 \Lambda_{\text {int }}$, contains 21 cells and 42 Hamamatsu R1355 PMTs that act as readout channels. The signal is sent to a shaper module, and then to CAEN V1724 flash ADCs. The KL performs electron-muon separation for the $0.5 \%$ of muons that decay. The ADC product, in which the left and right sides of one slab are multiplied and then divided by their average, is used to compensate for the effect of light attenuation. Figure 6 shows a larger ADC product average for electrons than for pions and muons.

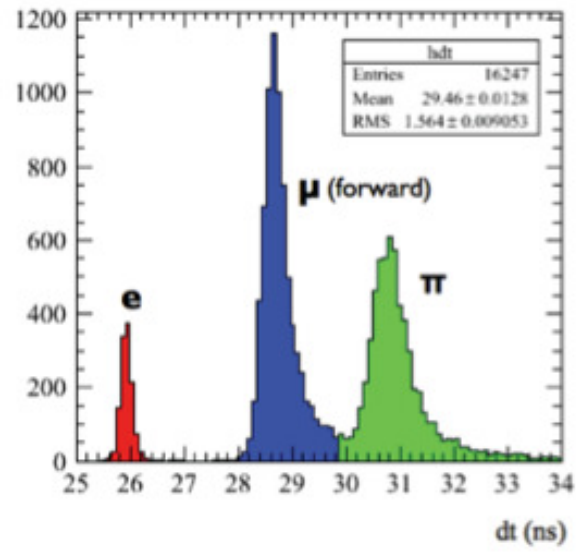

Figure 5: Time of flight separation for electrons, muons and pions for a $200 \mathrm{MeV} / \mathrm{c}, 6 \mathrm{~mm}$ rad beam.

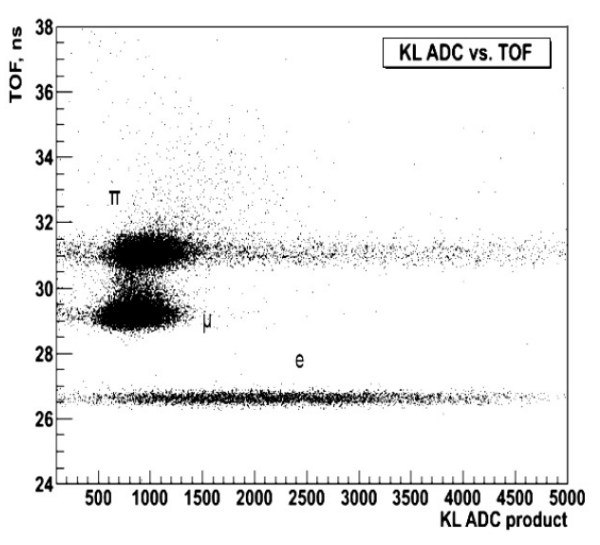

Figure 6: Time of flight measured by the TOF, versus the KL response as the ADC product.

The Electron Muon Ranger (EMR) consists of $1 \mathrm{~m}^{3}$ of extruded scintillator bars with wavelength shifting fibres, read out on both ends by Hamamatsu R7600-00-M64 multi-anode PMTs (Figure 7). There are $24 x / y$ modules (48 planes) with 59 bars per plane, for a total of 2832 bars of triangular cross-section and $1.7 \mathrm{~cm}$ thick [12]. Electrons give electromagnetic showers and muons give single tracks, whose energy can be measured by range up to $300 \mathrm{MeV} / \mathrm{c}$. The energy loss per plane can also be used to separate electrons from muons. A prototype was tested at RAL in the MICE muon beam. The full detector is under construction and will be installed in 2013. 


\subsection{MICE tracker}

Two scintillating fibre trackers have been constructed and have been fully characterised using cosmic rays (Figure 8) [13]. Each tracker will be embedded in a $4 \mathrm{~T}$ solenoidal field, which is currently being commissioned, and contains five scintillating fibre tracking stations, each consisting of three layers of $350 \mu \mathrm{m}$ diameter scintillating fibre doublets, read out by Visible Light Photon Counters (VLPCs), which are photon detectors with very high quantum efficiency $(80 \%)$. The resolution achieved in the cosmic-ray test stand was $661 \pm 2 \mu \mathrm{m}$, which includes multiple scattering, resulting in a channel resolution of $470 \mu \mathrm{m}$. The space-point hit efficiency is $99.8 \pm 0.1 \%$. The measured photoelectron mean yield is $11.23 \pm 0.01$ photoelectrons. The momentum resolution expected in the $4 \mathrm{~T}$ field is $1.1 \mathrm{MeV} / \mathrm{c}$ in transverse momentum and 3.9 MeV/c in longitudinal momentum.

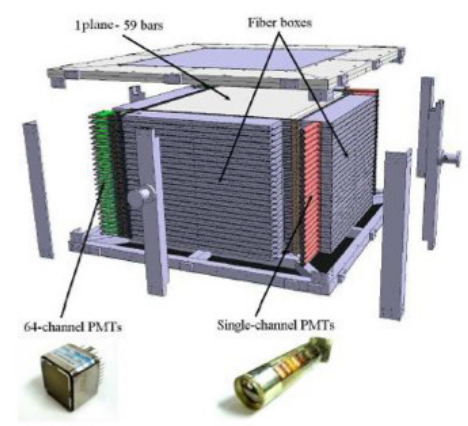

Figure 7: Schematic of Electron-Muon Ranger (EMR).

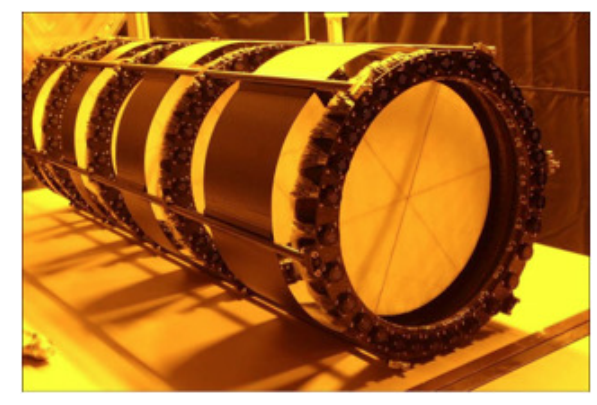

Figure 8: Scintillating fibre tracker under construction.

\section{Preliminary detector performance}

The MICE beam line in the Step I configuration was characterised during data-taking runs in 2010 and 2011 [14]. Nine different beam configurations, for positive and negative particles, covering $\varepsilon=3,6,10 \mathrm{~mm}$ rad and $p=140,200,240 \mathrm{MeV} / \mathrm{c}$ were selected. A preliminary measurement of emittance was carried out with the TOF system only $[15,16]$. The $(x, y)$ position of the muon and the angles $\left(x^{\prime}=p_{x} / p_{z}, y^{\prime}=p_{y} / p_{z}\right)$ were determined completely from measurements made by the TOF system, to determine the normalised emittance. Figure 4 shows the trace plots for a MICE beam, with $6 \mathrm{~mm}$ rad emittance and $p_{z}=200 \mathrm{MeV} / \mathrm{c}$, for both experimental data and Monte Carlo simulation. Once the trackers are installed, the emittance will be able to be determined with the required precision to carry out the MICE physics programme.

\section{Conclusions}

MICE Step I has been completed. The MICE beam, beam instrumentation and most of the particle identification detectors (three TOFs, two Cherenkov detectors and the KL preshower detector) have been constructed and commisioned. The EMR is currently under construction and willl be installed in 2013. The scintillating fibre tracker is completed but needs to be integrated with the tracker solenoid also in 2013. All these elements, in conjunction with the absorber-focus 

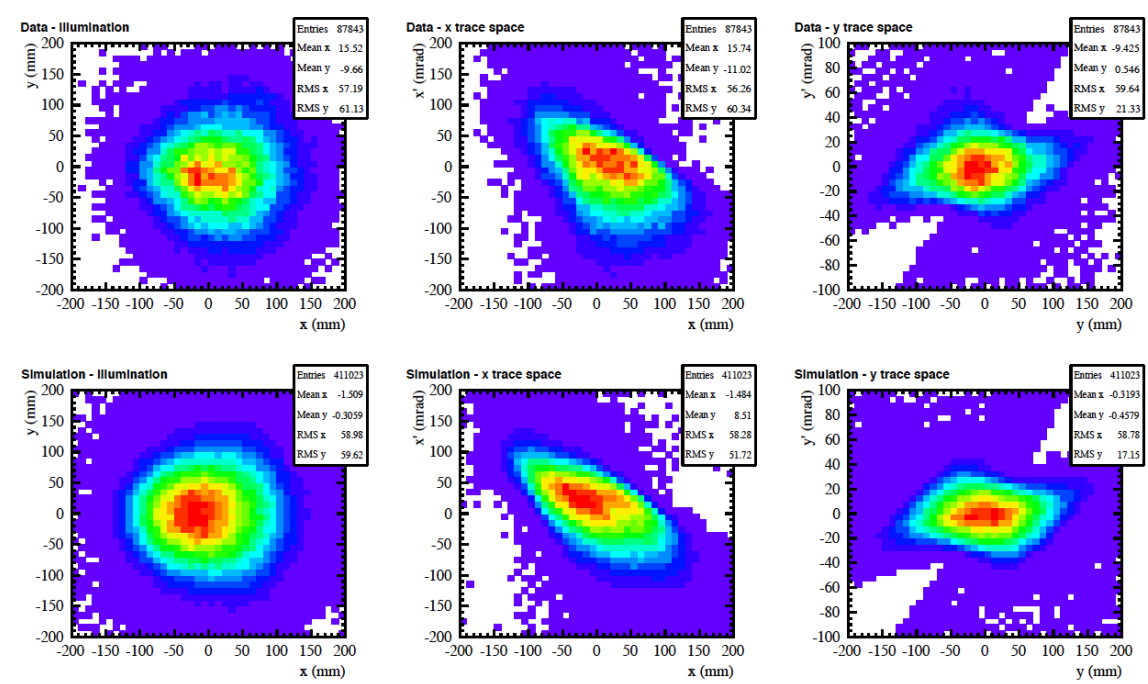

Figure 9: Emittance reconstruction in Step I of MICE for data (top) and Monte Carlo (bottom). Left: $y(\mathrm{~mm})$ vs $x(\mathrm{~mm})$. Middle: $x^{\prime}(\mathrm{mrad})$ vs $x(\mathrm{~mm})$. Right: $y^{\prime}(\mathrm{mrad})$ vs $y(\mathrm{~mm})$.

coil (AFC) modules make up MICE Step IV, with a physics programme that includes the measurement of multiple scattering and energy loss using solid $\mathrm{LiH}$ and liquid hydrogen absorbers. The installation of the RF Coupling Coil (RFCC) modules will allow the measurement of ionization cooling for the first time in the final Step VI of MICE.

\section{References}

[1] S. Choubey, et al., "IDS-NF, Interim Design Report", IDS-NF-20, arXiv:1112.2853 [hep-ex]

[2] F. P. An et al. [Daya-Bay Coll.], Phys. Rev. Lett. 108, 171803 (2012) [arXiv:1203.1669 [hep-ex].

[3] J. K. Ahn et al. [RENO Coll.], Phys. Rev. Lett. 108, 191802 (2012) [arXiv:1204.0626 [hep-ex].

[4] Y. Abe et al. [Double Chooz Coll.], Phys. Rev. Lett. 108, 131801 (2012) [arXiv:1112.6353 [hep-ex].

[5] K. Abe et al. [T2K Coll.], Phys. Rev. Lett. 107, 041801, 2011, arXiv:1106.2822 [hep-ex].

[6] A. Holin, PoS(EPS-HEP2011).

[7] F.J.P. Soler, PoS(ICHEP2012), 541.

[8] S. Geer, Ann. Rev. Nucl. Part. Sci. 59 (2009) 347.

[9] R. Bertoni et al., Nucl. Instr. and Meth. A615 (2010) 14.

[10] L. Cremaldi et al., IEEE Trans. Nucl. Sci. 56 (2009) 1475.

[11] A. Aloisio et al., [KLOE Coll.], Nucl. Inst. and Meth. A 494 (2002) 326.

[12] D. Lietti et al., Nucl. Instrum. Meth. A604 (2009) 314.

[13] M. Ellis et al., Nucl. Instrum. Meth. A659 (2011) 136.

[14] M. Bogomilov et al., [MICE Coll.], JINST 7 (2012) P05009.

[15] M.A.Rayner, PhD thesis, Oxford University, 2011.

[16] U. Bravar et al [MICE Coll.], arXiv:1110.1813 [physics.acc-ph] 